\title{
Malformations of the Posterior Fossa
}

\author{
${ }^{1}$ Vincenzo D'Addario, ${ }^{2}$ Luca Di Cagno, ${ }^{3}$ Pasquale Capuano, ${ }^{4}$ Mariangela Cialdella
}

\section{ABSTRACT}

Malformations of the posterior fossa include multiple entities which have been described in the recent years using different terminologies, thus causing some confusion in the literature about this topic.

They can be divided into two main categories: Cystic and noncystic. The cystic group includes Dandy-Walker malformation (DWM), vermian hypoplasia (VH), Blake's pouch cyst (BPC), mega cisterna magna (MCM), arachnoid cysts (ACs). The noncystic group includes cerebellar hypoplasia, rhombencephalosynapsis, Chiari II malformation.

The correct prenatal diagnosis allows to make the proper prognosis which is extremely variable ranging from cases with normal outcome to cases with severe neurodevelopmental delay.

The aim of this review is to evaluate the feasibility of sonographic diagnosis of the posterior fossa abnormalities and to discuss their clinical consequences.

Keywords: Blake pouch cyst, Cerebellum, Dandy Walker malformation, Megacisterna magna, Posterior fossa, Vermian hypoplasia

How to cite this article: D'Addario V, Di Cagno L, Capuano P, Cialdella M. Malformations of the Posterior Fossa. Donald School J Ultrasound Obstet Gynecol 2017;11(4):294-301.

Source of support: Nil

Conflict of interest: None

\section{INTRODUCTION}

Malformations of the posterior fossa include multiple entities which have been described in the recent years using different terminologies, thus causing some confusion in the literature about this topic. ${ }^{1,2}$

The aim of this review is to describe the sonographic appearance and clinical implications of the most common abnormalities of the posterior fossa.

\section{NORMAL SONOGRAPHIC ANATOMY OF THE POSTERIOR FOSSA}

Sonographic evaluation of the posterior fossa is routinely performed during the second-trimester scan to screen for fetal malformation. The basic examination is performed

\footnotetext{
${ }^{1}$ Professor, ${ }^{2,3}$ Consultant, ${ }^{4}$ Resident

${ }^{1,4}$ Department of Obstetrics and Gynecology, University of Bari Bari, Italy

${ }^{2}$ Obstetrics and Gynecology Unit, Cerignola Hospital, Italy

${ }^{3}$ Obstetrics and Gynecology Unit, Barletta Hospital, Italy

Corresponding Author: Vincenzo D'Addario, Professor Department of Obstetrics and Gynecology, University of Bari Bari, Italy, e-mail: daddariov@alice.it
}

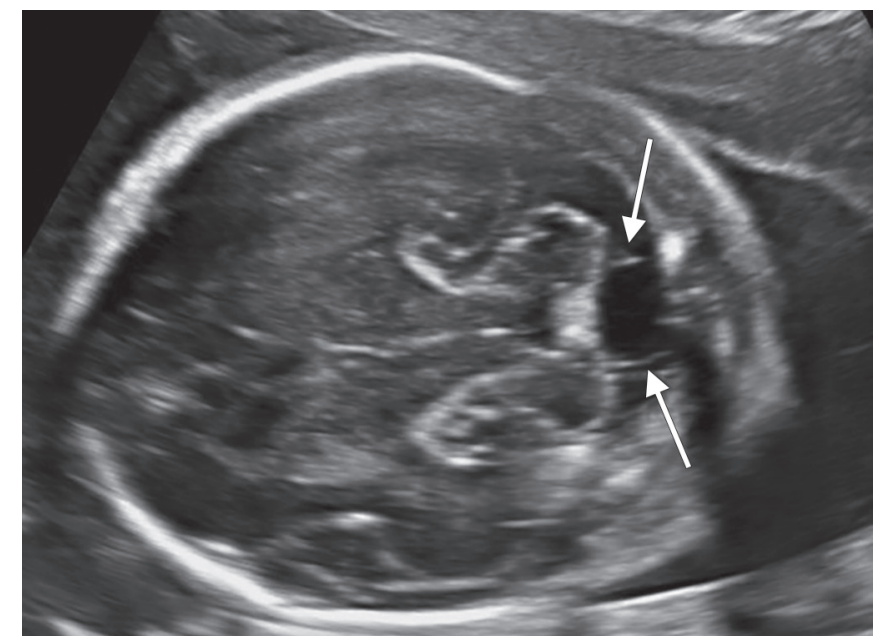

Fig. 1: Transcerebellar axial plane in a 21 weeks fetus. The cerebellar hemispheres and the interposed echogenic vermis are recognized, surrounded posteriorly by the anechoic cisterna magna. Posterior to the vermis, a small cystic structure is visible referred to Blake's pouch remnant (arrow)

on an axial plane which is slightly inferior and downward tilted in comparison with the transthalamic plane used for measuring biparietal diameter (BPD). In this transcerebellar plane, the cerebellar hemispheres and the interposed echogenic vermis are recognized; a small anechoic area referable to the fourth ventricle maybe seen in the anterior edge of the vermis. The anechoic cisterna magna is located behind the cerebellum and frequently shows thin septa referred to the falx cerebelli or a small cystic structure posterior to the vermis referred as Blake's pouch remnant (Fig. 1). ${ }^{3}$ On this plane, the cerebellar diameter and the cisterna magna width can be measured.

A complete evaluation of the posterior fossa requires the acquisition of the midsagittal plane. On this plane, the vermis shows a typical triangular shape located posteriorly to the pons and medulla; on the anterior border, an indentation is present due to the fastigium of the fourth ventricle; the cisterna magna surrounds the vermis posteriorly, and the tentorium is located superiorly (Fig. 2). The primary fissure may be seen dividing the upper third of the vermis from the inferior two-thirds. The midsagittal view may sometimes be difficult to obtain; in such cases, three-dimensional (3D) ultrasound may be helpful in reconstructing the midsagittal plane by the volume acquired starting by the axial one (Fig. 3 ). ${ }^{4}$ On the sagittal plane, multiple measurements may be obtained: Vermian height and width, ${ }^{5}$ brain stem-vermis angle (angle between the brain stem and the ventral face of the vermis), brain stem-tentorium angle (angle between the 


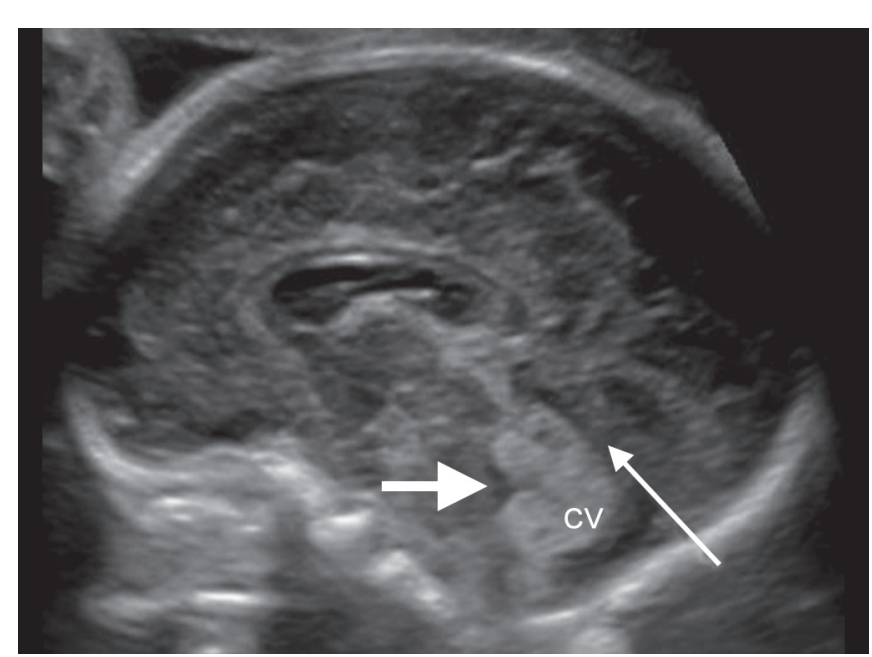

Fig. 2: Midsagittal plane of the posterior fossa: on this plane, the cerebellar vermis (CV) shows a typical triangular shape; on the anterior border an indentation is present due to the fastigium of the fourth ventricle (arrowhead); the cisterna magna surrounds the vermis posteriorly and the tentorium is located superiorly (arrow)

tentorium and the brain stem). ${ }^{6,7}$ These measurements may be useful in the differential diagnosis of different cystic malformations of the posterior fossa.

\section{CLASSIFICATION OF THE POSTERIOR FOSSA MALFORMATIONS}

Different classifications of the posterior fossa abnormalities have been proposed. The embryological and etiological classifications are complex, incomplete, and not easily related to the ultrasonic findings. ${ }^{8}$ It is more useful to refer to morphological classifications. Different morphological classifications have been suggested..$^{9-13}$ The easiest way to classify the posterior fossa malformation is to divide them into two main groups: The cystic and the noncystic anomalies.

The cystic anomalies include

- Dandy Walker Malformation (DWM)

- Vermian hypoplasia $(\mathrm{VH})$

- Blake's pouch cyst (BPC)

- Megacisterna magna (MCM)

- Arachnoid cysts (ACs)

The noncystic anomalies include

- Cerebellar hypoplasia

- Rhombencephalosynapsis

- Chiari II malformation
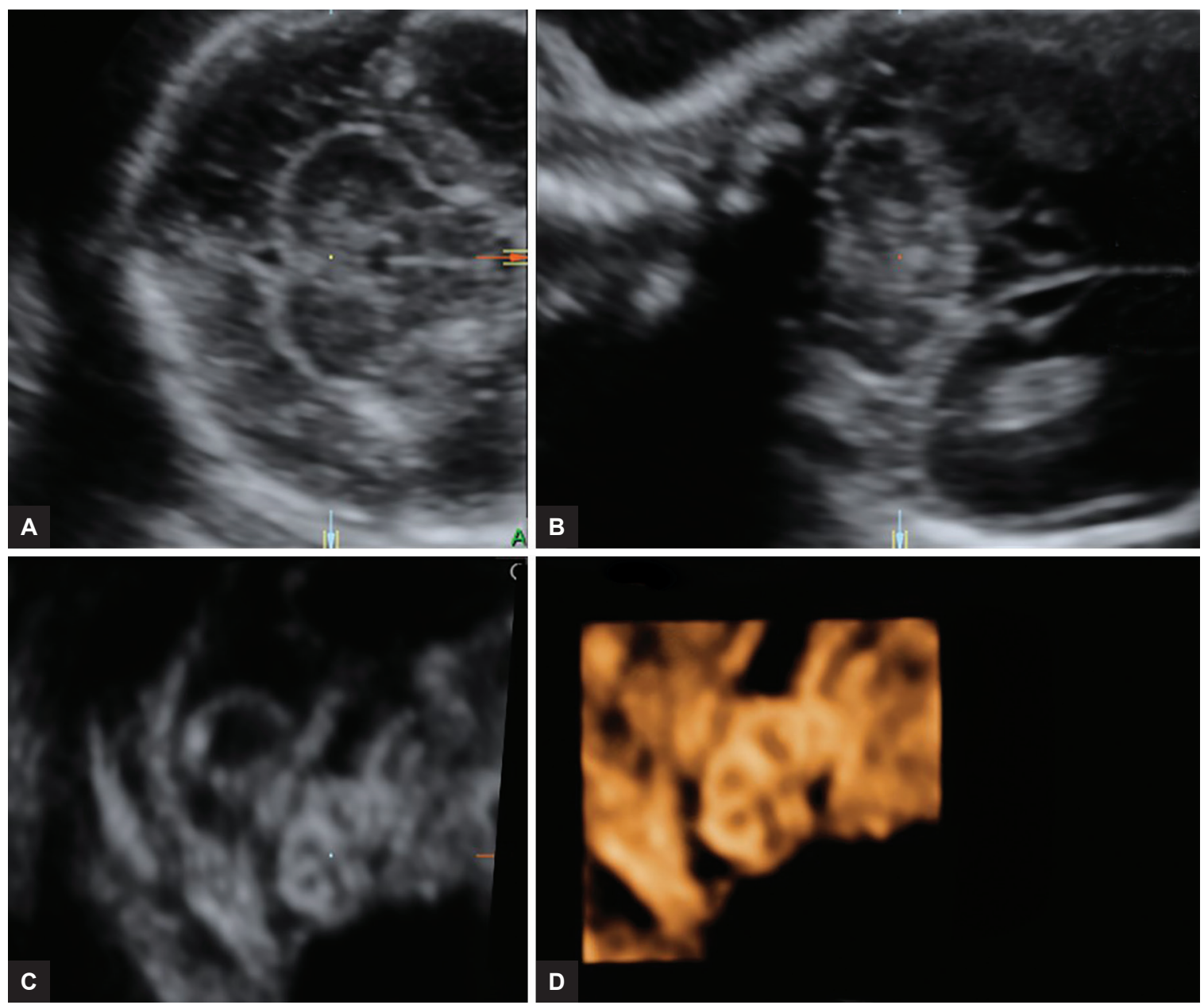

Figs 3A to D: Three-dimensional evaluation of the posterior fossa: The midsagittal view is reconstructed by a volume acquired starting by the axial one 

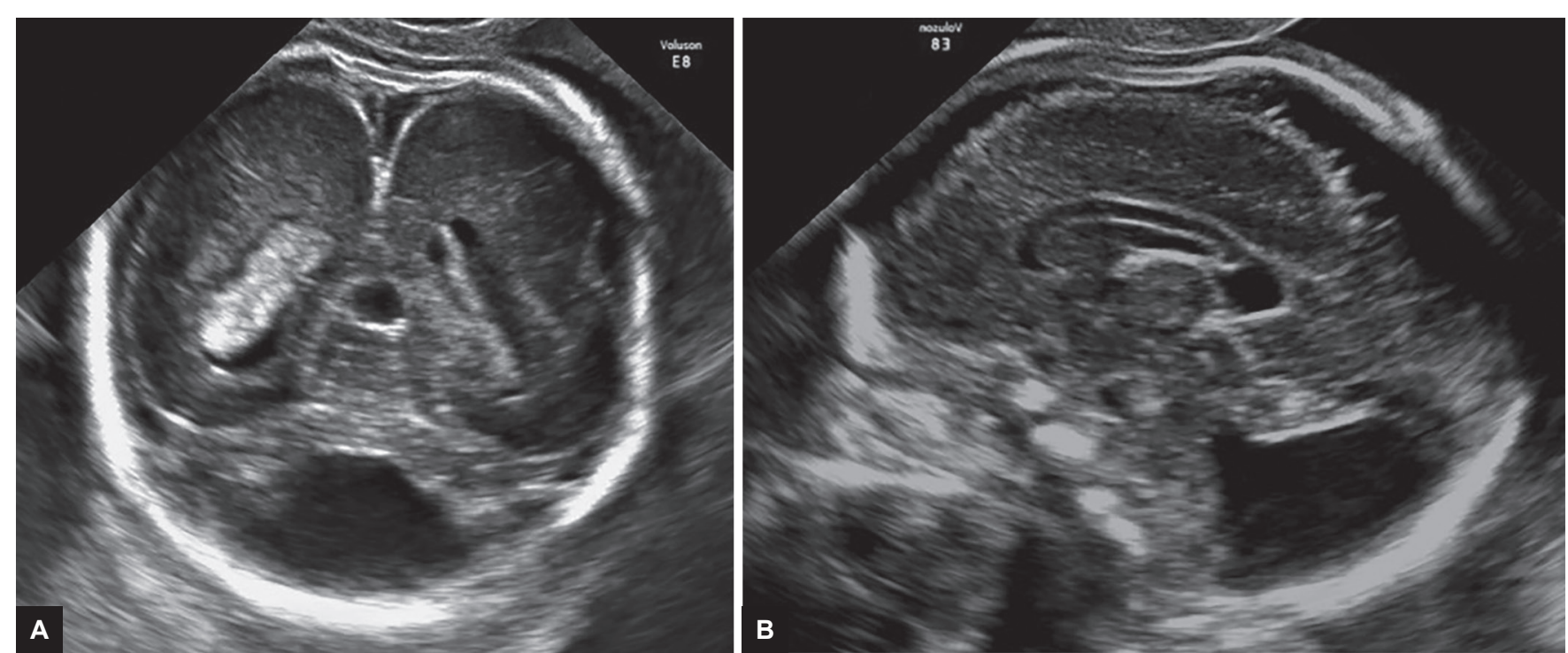

Figs 4A and B: Dandy-Walker malformation: (A) The axial plane with a huge cyst in the posterior fossa displacing the cerebellar hemispheres laterally; and (B) the midsagittal plane with the anticlockwise rotation of the hypoplastic vermis and the upward displacement of the tentorium

\section{CYSTIC ANOMALIES}

\section{Dandy-Walker Malformation}

This malformation derives from an abnormal development of the rhombencephalic roof involving the anterior membranous area. It is characterized by complete or partial agenesis and upward rotation of the cerebellar vermis, cystic dilatation of the fourth ventricle, and wide posterior fossa with upward displacement of the tentorium.

Sonography shows in the axial plane the presence of a huge cyst in the posterior fossa displacing the cerebellar hemispheres laterally. The midsagittal plane shows the anticlockwise rotation of the hypoplastic vermis and the upward displacement of the tentorium (Fig. 4). The brain stem-vermis angle is high (usually more than $45^{\circ}$ ). ${ }^{7}$

Accordingly, with a recent meta-analysis by $\mathrm{D}^{\prime}$ Antonio et al, ${ }^{14} \mathrm{DWM}$ was associated with other central nervous system (CNS) and extra-CNS anomalies in 60.9 and $42.6 \%$ of cases respectively. Ventriculomegaly was a common finding detected prenatally in $31.3 \%$; it is related to dynamic changes in the cerebrospinal fluid, secondary to the mass effect of the cystic malformation, and after birth the association rises to $68 \% .{ }^{15}$ The prevalence of additional CNS abnormalities missed at ultrasound and detected only on prenatal magnetic resonance imaging (MRI) was $13.7 \%$, while the rates of additional CNS and extra-CNS anomalies missed at prenatal imaging by either ultrasound or MRI and detected only after birth were 18.2 and $18.9 \%$ respectively. The prenatal diagnosis of DWM was not confirmed after birth in $28.2 \%$ of cases: The misdiagnoses proved to be BPC, VH, Joubert syndrome, or even normal cases.
Chromosomal abnormalities are frequently associated. In the meta-analysis by D'Antonio et al ${ }_{1}^{14}$ the prevalence in isolated cases was $16.3 \%$, with chromosomal deletions representing the most common anomaly. In the presence of associated CNS and extra-CNS anomalies, the association rises to $35 \%$. The association with nonchromosomal genetic syndromes (Walker-Warburg, Aicardi, Neu-Laxova) is also possible.

The prognosis is usually poor with different degrees of neurodevelopmental delay in cases with associated CNS anomalies and abnormal karyotype. In the isolated cases, the overall rate of abnormal neurodevelopmental status, according to the meta-analysis by $\mathrm{D}^{\prime}$ Antonio et $\mathrm{al}_{1}{ }^{15}$ was $58.2 \%$. The neurodevelopmental delay includes different degrees of severity in motor control and language impairment. Ventriculomegaly requiring a ventriculoperitoneal shunt to reduce raised intracranial pressure occurred in $62.7 \%$ of the cases.

\section{Vermian Hypoplasia}

This term describes a condition characterized by the presence of a small vermis in a normally sized posterior fossa. The hypoplasia usually affects the inferior portion of the vermis. This condition was defined in the past as Dandy-Walker variant. It derives from an abnormal development of the rhombencephalic roof involving the anterior membranous area.

The sonographic diagnosis of $\mathrm{VH}$ is not easy: Falsepositive and false-negative cases may occur. In the axial plane, a small cleft is present between the cerebellar hemispheres. The midsagittal plane shows a vermis smaller than normal for hypoplasia of the inferior portion; it is moderately anticlockwise rotated (less than $45^{\circ}$ ) with an 

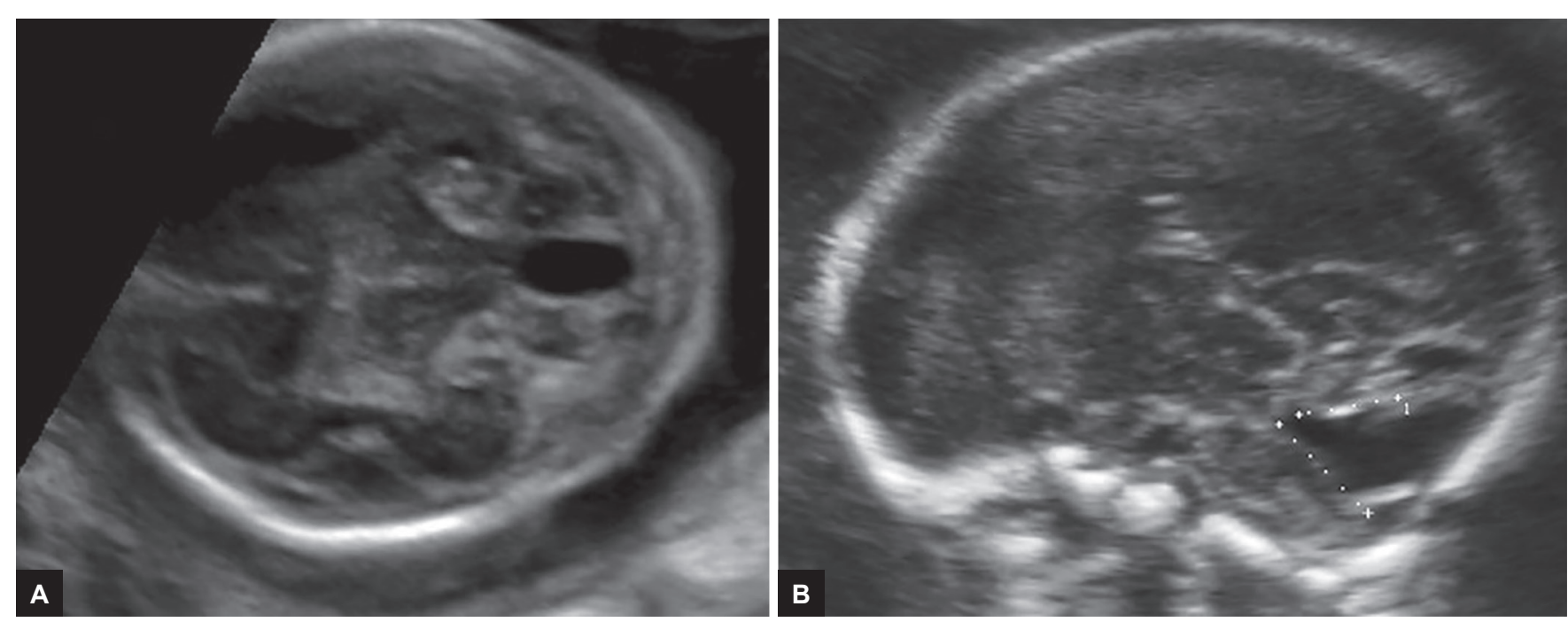

Figs 5A and B: Vermian hypoplasia: The axial plane (A) shows a small cleft between the cerebellar hemispheres. The midsagittal plane (B) shows a vermis smaller than normal for hypoplasia of the inferior portion with a moderate anticlockwise rotation (less than $45^{\circ}$ ) and with an apparent communication of the fourth ventricle with the cisterna magna. The posterior fossa size and the tentorium insertion are normal

apparent communication of the fourth ventricle with the cisterna magna. ${ }^{7}$ The posterior fossa size and the tentorium insertion are normal (Fig. 5).

The $\mathrm{VH}$ is frequently associated with other anomalies. In the meta-analysis by $\mathrm{D}^{\prime}$ Antonio et $\mathrm{al}_{1}^{15}$ the rates of associated CNS and extra-CNS anomalies were 56.1 and $49.2 \%$ respectively. The percentage of chromosomal abnormalities was $3.3 \%$ (only one chromosomal deletion among 30 fetuses tested). The proportion of CNS anomalies detected only after delivery was $14.2 \%$. The prenatal diagnosis was not confirmed after delivery in $32.4 \%$ of the cases, which resulted to be normal in 9 out of 10 , thus confirming the high risk of false positive of prenatal diagnosis.

The prognosis is usually poor in cases with associated CNS anomalies and chromosomopaties. In the isolated cases, the outcomes reported in the literature are extremely heterogeneous with reported neurodevelopmental delay ranging from 0 to $100 \%$. The largest series is reported by Tarui et $\mathrm{al}^{16}$ : On 20 fetuses with prenatal diagnosis of $\mathrm{VH}$ on MRI, 12 proved to be isolated after birth and all of them had normal neurodevelopmental outcome.

\section{Blake's Pouch Cyst}

Blake's pouch cyst is a normal transient condition during the development of the posterior fossa. It is an evagination of the roof of the fourth ventricle protruding posteriorly below the developing cerebellar vermis. At approximately 10 weeks, it perforates forming the foramen of Magendie opening in the cisterna magna, which in the same time is developed by cavitation of the primitive meninx. The lack of fenestration causes a cystic dilatation of the pouch that becomes a true cyst protruding into the cisterna magna and causing a lifting and anticlockwise rotation of a normally developed cerebellar vermis. ${ }^{17}$ Actually, it originates from an abnormal development of the posterior membranous area and is also defined as persistent Blake's pouch. $^{18}$

In the axial plane, a small "keyhole"-shaped cleft between the two cerebellar hemispheres is seen. The midsagittal plane shows a slight anticlockwise rotation (less than $30^{\circ}$ ) of a normally developed vermis. ${ }^{7}$ It is sometimes possible to recognize the thin roof of the cyst inside the cisterna magna (Fig. 6). ${ }^{16}$

The rates of associated CNS and extra-CNS anomalies are 11.5 and $25.3 \%$ respectively, according to the already mentioned meta-analysis by $\mathrm{D}^{\prime}$ Antonio et al. ${ }^{14}$ The same study reported only one case of aneuploidy among 45 fetuses tested; no associated CNS or extra-CNS anomalies were detected only after birth; the prenatal diagnosis was not confirmed after birth in $9.8 \%$ of the cases (one $\mathrm{AC}$, one MCM, one normal).

In almost half of the cases, BPC may disappear in the late second trimester as a consequence of late fenestration or late opening of the Luschka apertures. This event usually occurs at 14 to 17 weeks, but late aperture at around 26 weeks is possible. ${ }^{19}$

The neurological outcome of fetuses with BPC is usually good since BPC derives from the posterior membranous area and does not contain neurons: In the largest series by Gandolfi Colleoni et $\mathrm{al}^{20}$ of 20 fetuses with BPC, only 1 showed mild psychomotor disorder at 3 years. Ventriculomegaly may appear in some cases $(12.4 \%$ of the cases in the meta-analysis). ${ }^{15}$

\section{Mega Cisterna Magna}

It is a condition characterized by a wide cisterna magna and a normally developed cerebellum. It is the 

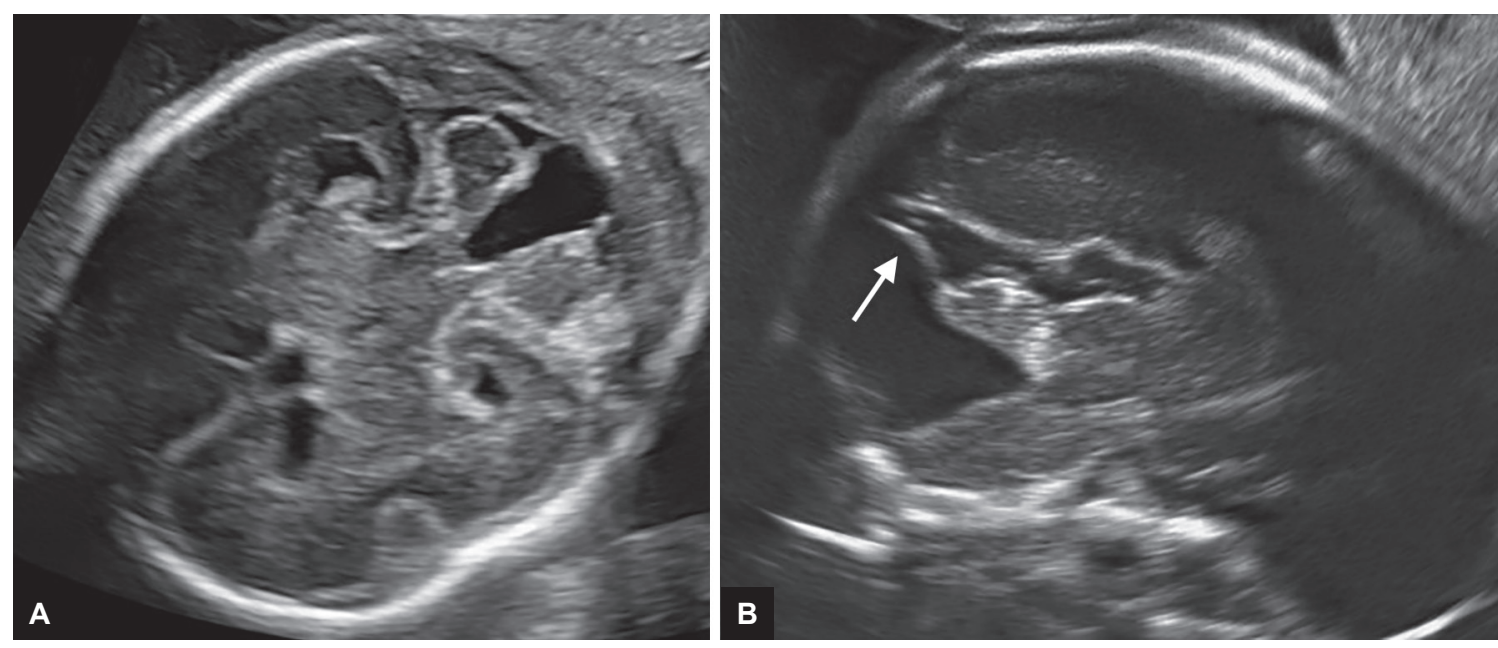

Figs 6 A and B: Blake's pouch cyst: In the axial plane (A) a "keyhole" cleft between the two cerebellar hemispheres is seen. The midsagittal plane; and $(B)$ with a slight anticlockwise rotation of a normally developed vermis. It is possible to recognize the thin roof of the cyst inside the cisterna magna (arrow)
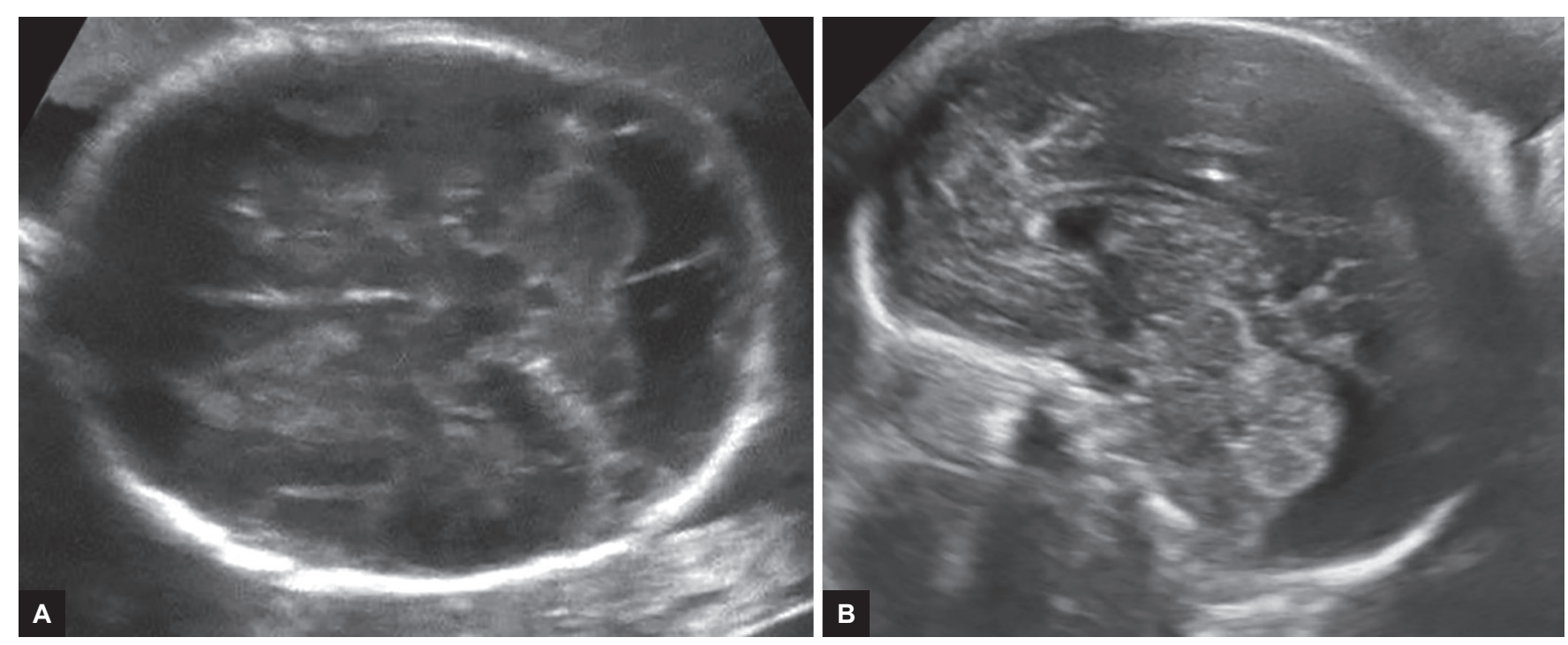

Figs 7A and B: Mega cisterna magna: The axial transcerebellar plane $(A)$ represents a large cisterna magna with an anterior to posterior diameter $>10 \mathrm{~mm}$. The midsagittal plane; and $(B)$ with normal vermis and normal fourth ventricle

consequence of an abnormal development of the posterior membranous area maybe due to a late fenestration of Blake's pouch which enlarges until it fills the cisterna magna completely.

The sonographic diagnosis is feasible in the axial transcerebellar plane showing a large cisterna magna with an anterior to posterior diameter $>10 \mathrm{~mm}$. The midsagittal plane shows normal vermis and normal fourth ventricle (Fig. 7).

The association with CNS (mainly ventriculomegaly) and extra-CNS anomalies is 12.6 and $16.6 \%$ respectively. ${ }^{14}$ The association with aneuploidy is extremely low. The neurological outcome is usually good in isolated cases. Mild degrees of neurodevelopmental delay were found in $13.8 \%$ of the cases ${ }^{15}$ : Mainly mild language disorders, visuospatial perceptions and attention problems, and mild abnormal motor development.

\section{Arachnoid Cyst}

It is a cystic collection of cerebrospinal fluid not communicating with ventricular system and with the surrounding arachnoid spaces.

The sonographic appearance is that of a retrocerebellar anechoic fluid collection which pushes and displaces a normally developed cerebellum (Fig. 8). Huge cyst may obstruct cerebrospinal fluid flow causing ventriculomegaly.

The prognosis is usually good. Huge cysts may require surgery after delivery. ${ }^{21}$

\section{NONCYSTIC ANOMALIES}

\section{Cerebellar Hypoplasia}

In the total $\mathrm{CH}$, the whole cerebellum, both vermis, and hemispheres are hypoplastic (Fig. 9). It is usually 


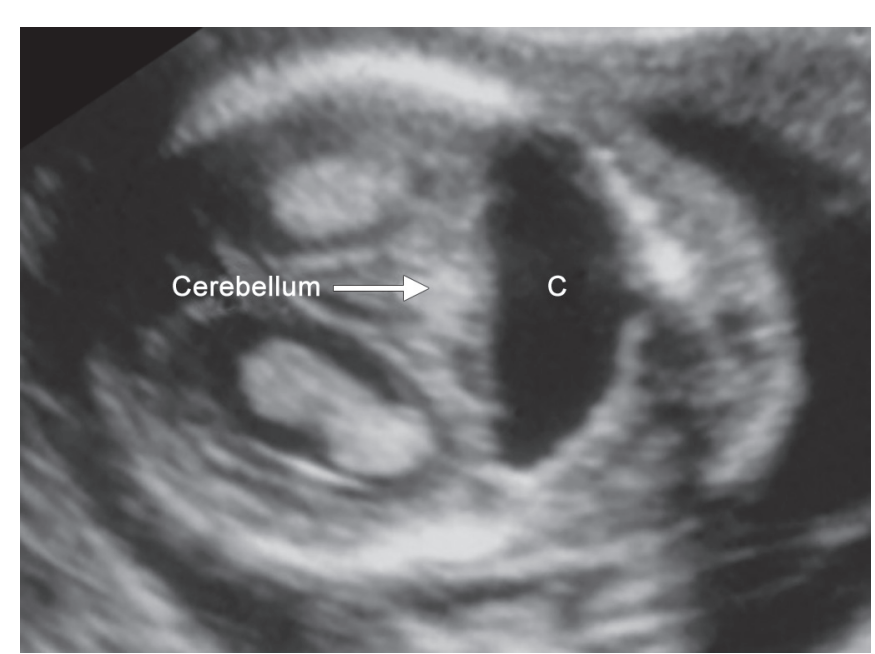

Fig. 8: Posterior fossa AC: The axial transcerebellar plane with a retro-cerebellar anechoic fluid collection (C) which pushes and displaces a normally developed cerebellum (arrow)

part of more complex malformations affecting also the brain stem and the medulla (pontocerebellar hypoplasia) and the cerebral cortex (lissencephaly) or may be part of syndromes with extra-CNS anomalies. It has also been reported in fetuses affected by Zika virus infection. ${ }^{22}$

The prognosis is poor with severe neurodevelopmental delay.

The $\mathrm{CH}$ may also be focal affecting only one hemisphere (unilateral cerebellar hypoplasia; Fig. 10). It may be idiopathic or secondary to hemorrhage and/or ischemic insult, suggesting a clastic origin, particularly when imaging follow-up reveals changes over time..$^{23}$ It may be part of PHACE syndrome (posterior fossa anomalies, hemangioma, arterial anomalies, cardiac abnormalities, eye abnormalities).

The amount of surface loss of cerebellar hemisphere does not correlate with poor prognosis. Unilateral cerebellar hypoplasia with normal vermis is often associated with normal outcome.

\section{Rhombencephalosynapsis}

This rare anomaly is characterized by complete or partial absence of the cerebellar vermis, with varying degrees of midline fusion of the cerebellar hemispheres. ${ }^{24}$

In the axial transcerebellar plane, the cerebellum is small and dysmorphic due to the absence of the vermis with fusion of the cerebellar hemispheres (Fig. 11). The posterior fossa is small.

This anomaly is frequently associated with other sovratentorial CNS malformations and usually carries a severe prognosis.

\section{Chiari II Malformation}

It is a complex anomaly characterized by descent and elongation of the cerebellar vermis through the foramen magnum, descent and kinking of the brain stem, small posterior fossa, and low insertion of the tentorium. It is associated with spinal dysraphism, which is the primary cause of the posterior fossa maldevelopment. ${ }^{25}$ Ventriculomegaly, polygyria, cortical heterotopia, and dysgenesis of the corpus callosum may be associated.

In the axial plane, the cerebellum shows the typical "banana" shape, and the cisterna magna is effaced. In the sagittal plane, the small and "crowded" posterior fossa may be recognized (Fig. 12). The severity of the posterior fossa hypoplasia may be evaluated by measuring the angle formed by the clivus and the occipital bone, ${ }^{26}$ or the area delimited by the clivus, the occipital bone, and the tentorium. ${ }^{27}$

The prognosis mainly depends on the level and severity of the spinal defect and on the presence of associated ventriculomegaly and brain abnormalities.
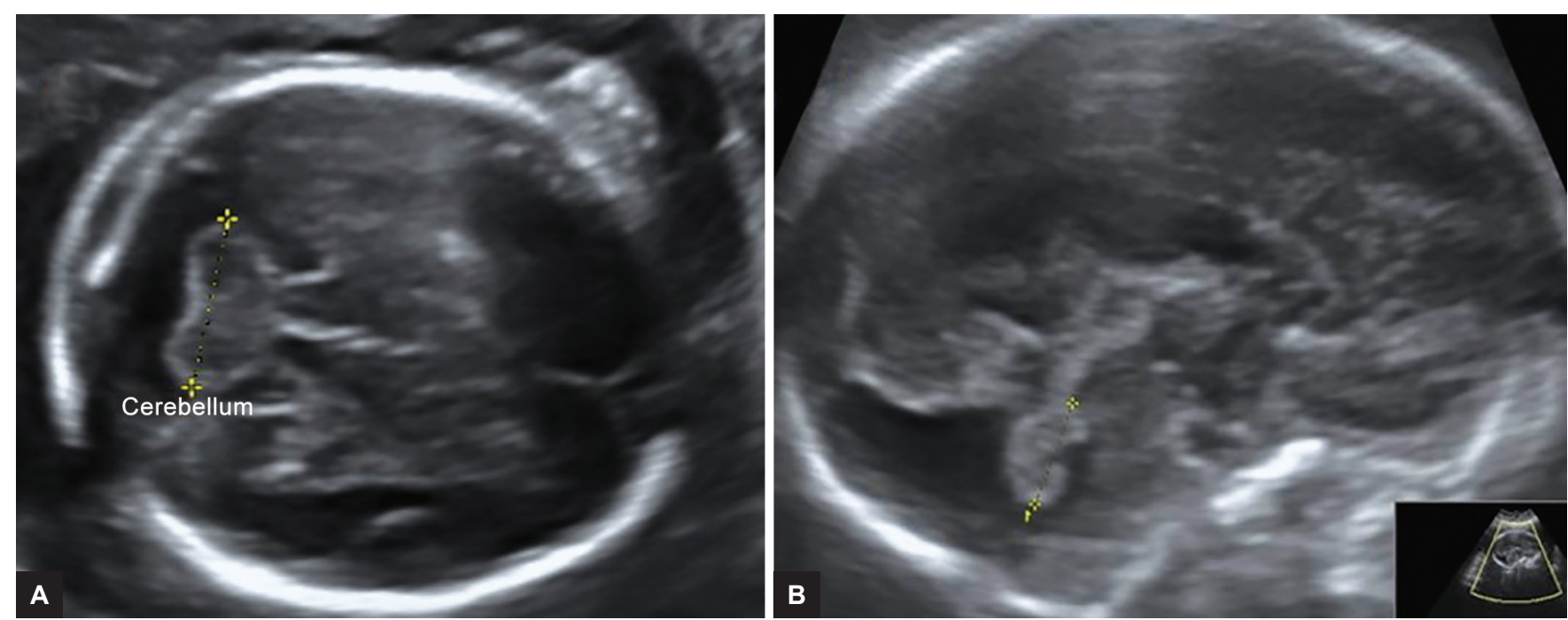

Figs 9A and B: Cerebellar hypoplasia: The whole cerebellum, both $(A)$ hemispheres; and $(B)$ vermis are hypoplastic 


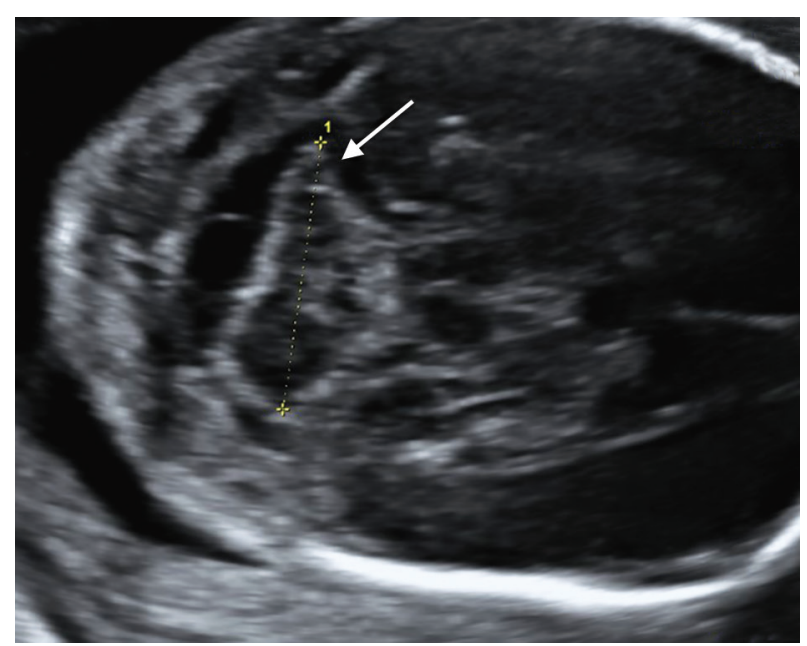

Fig. 10: Focal cerebellar hypoplasia: Only one cerebellar hemisphere is hypoplastic. The vermis is normal

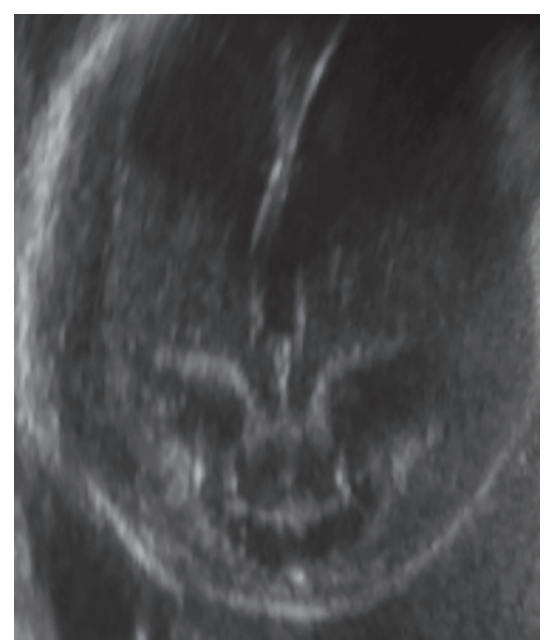

Fig. 11: Rhombencephalosynapsis: In the axial transcerebellar plane, the cerebellum is small and dysmorphic due to the absence of the vermis with fusion of the cerebellar hemispheres
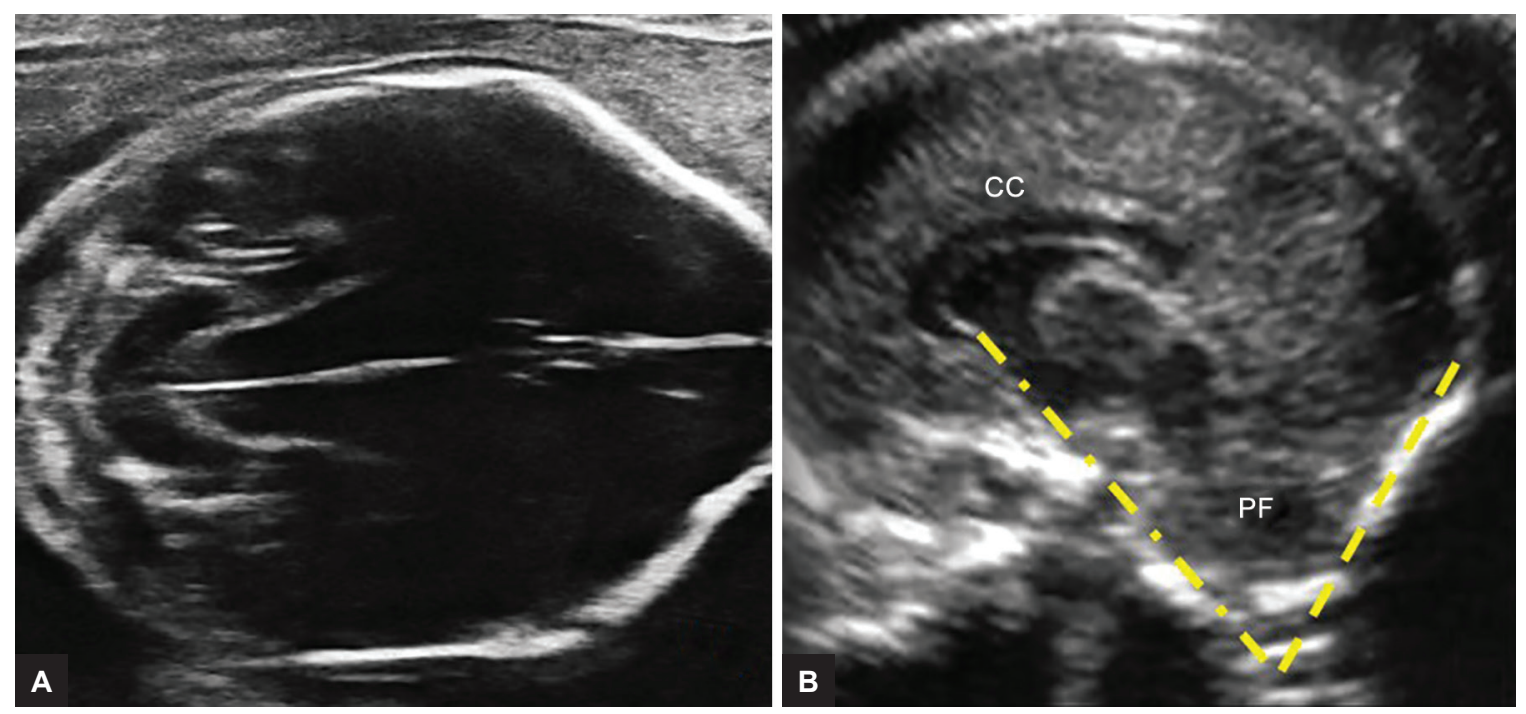

Figs 12A and B: Chiari II malformation: In the axial plane (A) the cerebellum shows the typical "banana" shape, and the cisterna magna is effaced. In the sagittal plane $(B)$, the small and "crowded" posterior fossa may be recognized

\section{REFERENCES}

1. Garel C, Fallet-Bianco C, Guibaud L. The fetal cerebellum: development and common malformations. J Child Neurol 2011 Dec;26(12):1483-1492.

2. Robinson AJ. Inferior vermian hypoplasia-preconception, misconception. Ultrasound Obstet Gynecol 2014 Feb;43(2):123-136.

3. Robinson AJ, Goldstein R. The cisterna magna septa: vestigial remnants of Blake's pouch and a potential new marker for normal development of the rhombencephalon. J Ultrasound Med 2007 Jan;26(1):83-95.

4. Viñals F, Muñoz M, Naveas R, Shalper J, Giuliano A. The fetal cerebellar vermis: anatomy and biometric assessment using volume contrast imaging in the C-plane (VCIC). Ultrasound Obstet Gynecol 2005 Nov;26(6):622-627.

5. Zalel Y, Seidman DS, Brand N, Lipitz S, Achiron R. The development of the fetal vermis: an in-utero sonographic evaluation. Ultrasound Obstet Gynecol 2002 Feb;19(2):136-139.

6. Paladini D, Volpe P. Posterior fossa and vermian morphometry in the characterization of fetal cerebellar abnormalities: a prospective three-dimensional ultrasound study. Ultrasound Obstet Gynecol 2006 May;27(5):482-489.

7. Volpe P, Contro E, De Musso F, Ghi T, Farina A, Tempesta A, Volpe G, Rizzo N, Pilu G. Brainstem-vermis and brainstem-tentorium angles allow accurate categorization of fetal upward rotation of cerebellar vermis. Ultrasound Obstet Gynecol 2012 Jun;39(6):632-635.

8. Parisi MA, Dobyns WB. Human malformations of the midbrain and hindbrain: review and proposed classification scheme. Mol Genet Metab 2003 Sep-Oct;80(1-2):36-53.

9. Barkovich, A. Congenital malformations of the brain and skull. In: Barkovich A, editor. Pediatric neuroradiology. 3rd ed. Philadelphia (PA): Lippincott Williams; 2000. pp. 251-381.

10. Tortori-Donati, P. Pediatric neuroradiology. New York: Springer; 2005.

11. Guibaud L, des Portes V. Plea for an anatomical approach to abnormalities of the posterior fossa in prenatal diagnosis. Ultrasound Obstet Gynecol 2006 Apr;27(5):477-481.

12. Patel S, Barkovich AJ. Analysis and classification of cerebellar malformations. AJNR Am J Neuroradiol 2002 Aug;23(7): 1074-1087. 
13. Chapman T, Mahalingam S, Ishak GE, Nixon JN, Siebert J, Dighe MK. Diagnostic imaging of posterior fossa anomalies in the fetus and neonate: Part 1, normal anatomy and classification of anomalies. Clin Imaging 2015 Jan-Feb;39(1):1-8.

14. D'Antonio F, Khalil A, Garel C, Pilu G, Rizzo G, Lerman-SagieT, Bhide A, Thilaganathan B, Manzoli L, Papageorghiou AT. Systematic review and meta-analysis of isolated posterior fossa malformations on prenatal ultrasound imaging (part 1): nomenclature, diagnostic accuracy and associated anomalies. Ultrasound Obstet Gynecol 2016 Jun;47(6):690-697.

15. D'Antonio F, Khalil A, Garel G, Pilu G, Rizzo N, Lerman-Sagie T, Bhide A, Thilaganathan B, Manzoli L, Papageorghiou AT. Systematic review and meta-analysis of isolated posterior fossa malformations on prenatal imaging (part 2): neurodevelopmental outcome. Ultrasound Obstet Gynecol 2016 Jul;48(1):28-37.

16. Tarui T, Limperopoulos C, Sullivan NR, Robertson RL, du Plessis AJ. Long-term developmental outcome of children with a fetal diagnosis of isolated inferior vermian hypoplasia. Arch Dis Child Fetal Neonatal Ed 2014 Jan;99(1):F54-F58.

17. Paladini D, Quarantelli M, Pastore G, Sorrentino M, Sglavo G, Nappi C. Abnormal or delayed development of the posterior membranous area of the brain: anatomy, ultrasound diagnosis, natural history and outcome of Blake's pouch cyst in the fetus. Ultrasound Obstet Gynecol 2012 Mar;39(3):279-287.

18. Tortori-Donati P, Fondelli MP, Rossi A, Carini S. Cystic malformations of the posterior fossa originating from a defect of the posterior membranous area. Mega cisterna magna and persisting Blake's pouch: two separate entities. Childs Nerv Syst 1996 Jun;12(6):303-308.

19. Brocklehurst G. The development of the human cerebrospinal fluid pathway with particular reference to the roof of the fourth ventricle. J Anat 1969 Nov;105(Pt 3):467-475.
20. GandolfiColleoniG, Contro E, CarlettiA, Ghi T,Campobasso G, Rembouskos G, Volpe G, Pilu G, Volpe P. Prenatal diagnosis and outcome of fetal posterior fossa fluid collections. Ultrasound Obstet Gynecol 2012 Jun;39(6):625-631.

21. De Keersmaecker B, Ramaekers P, Claus F, Witters I, Ortibus E, Naulaers G, Van Calenbergh F, De Catte L. Outcome of 12 antenatally diagnosed fetal arachnoid cysts: case series and review of the literature. Eur J Paediatr Neurol 2015 Mar;19(2): 114-121.

22. Meneses JDA, Ishigami AC, de Mello LM, de Albuquerque LL, de Brito CAA, Cordeiro MT, Pena LJ. Lessons learned at the epicenter of Brazil's congenital Zika epidemic: evidence from 87 confirmed cases. Clin Infect Dis 2017 May;64(10): 1302-1308

23. Massoud M, Cagneaux M, Garel C, Varene N, Moutard ML, Billette T, Benezit A, Rougeot C, Jouannic JM, Massardier J, et al. Prenatal unilateral cerebellar hypoplasia in a series of 26 cases: significance and implications for prenatal diagnosis. Ultrasound Obstet Gynecol 2014 Oct;44(4):447-454.

24. Poretti A, Boltshauser E. Fetal diagnosis of rhombencephalosynapsis. Neuropediatrics 2015 Dec;46(6):357-358.

25. McLone DG, Knepper PA. The cause of Chiari II malformation: a unified theory. Pediatr Neurosci 1989 Feb;15(1):1-12.

26. D'Addario V, Pinto V, Del Bianco A, Di Naro E, Tartagni M, Miniello G, Serio G. The clivus-supraocciput angle: a useful measurement to evaluate the shape and size of the fetal posterior fossa and to diagnose Chiari II malformation. Ultrasound Obstet Gynecol 2001 Aug;18(2):146-149.

27. Leibovitz Z, Haratz KK, Malinger G, Shapiro I, Pressman C. Fetal posterior fossa dimensions: normal and anomalous development assessed in mid-sagittal cranial plane by threedimensional multiplanar sonography. Ultrasound Obstet Gynecol 2014 Feb;43(2):147-153. 